\title{
The Height Gain in Scoliotic Deformity Correction: Assessed by New Predictive Formula
}

\author{
Ahmet Yılmaz Şarlak, ${ }^{1}$ Halil Atmaca, ${ }^{2}$ Resul Musaoğlu, ${ }^{1}$ and Elşen Veli Veliev ${ }^{3}$ \\ ${ }^{1}$ Department of Orthopaedics and Traumatology, School of Medicine, Kocaeli University, Umuttepe Central Campus, \\ 41380 Umuttepe, Kocaeli, Turkey \\ ${ }^{2}$ Department of Orthopaedics and Traumatology, Midyat State Hospital, Mardin, Turkey \\ ${ }^{3}$ Physics Department, Art and Science Faculty, Kocaeli University, Kocaeli, Turkey \\ Correspondence should be addressed to Ahmet Yılmaz Şarlak, kouortopcom@yahoo.com
}

Received 19 January 2012; Revised 25 February 2012; Accepted 14 March 2012

Academic Editor: Hugo Palmans

Copyright (๑) 2012 Ahmet Yllmaz Şarlak et al. This is an open access article distributed under the Creative Commons Attribution License, which permits unrestricted use, distribution, and reproduction in any medium, provided the original work is properly cited.

\begin{abstract}
Height gain after scoliosis correction is of a special interest for the patient and family. Ylikoski was the first to suggest a formula predicting height loss in untreated scoliotic patients. Stokes has recently suggested a new formula by using Cobb angle to determine height loss in idiopathic curves. We hypothesized that new additional variables to Cobb angle such as apical vertebral translation (AVT), number of instrumented segments $(N)$, and disc heights may increase the accuracy of predicted height gain. According to our findings simple expression for height gain by simplified version of the formula is: $S P \Delta H=0.0059 X_{1} \theta_{1}+2.3\left(1-\left(\theta_{2} / \theta_{1}\right)\right) N$, where $\theta_{1}$ is preoperative Cobb angle, $X_{1}$ is preoperative AVT, $\theta_{2}$ is postoperative Cobb angle, and $N$ is the number of instrumented vertebra. The purpose of this study is to analyze a new mathematical formula to predict height gain after scoliotic deformity correction.
\end{abstract}

\section{Introduction}

A spine of a given length will have a lesser vertical height when curved, hence scoliosis is associated with height loss [1]. With the increasing importance of cosmetic concerns, short stature might be an important element of body dissatisfaction especially in adolescent patients with scoliotic deformity. There have been previous formulas curvilinearly correlating trunk height loss to the angulation of the primary curve. None of these formulas, however, had been confirmed clinically with respect to height gain in surgically corrected curves to our knowledge [1-4].

The purpose of this study is to analyze a new formula to predict height gain after scoliotic deformity correction taking into account the contribution of new variables (apical vertebral translation (AVT), number of instrumented segments $(N)$, and disc heights) to increase accuracy which has not been used on previous formulas.

\section{Materials and Methods}

Thirty-six patients (30 females and 6 males) with single idiopathic curve treated by posterior instrumentation and fusion were analyzed. The mean age of the patients was 16.7 years (range. 7-45). The curves were classified according to Lenke classification system. Nineteen were type $1 \mathrm{~A}$, three type $1 \mathrm{~B}$, five type $1 \mathrm{C}$, and nine type $5 \mathrm{C}$. The heights of the patients were measured preoperative one week after surgery by the same resident who is unrelated to this study, and the difference between preoperative and postoperative body height noted as $\Delta H(\mathrm{~mm})$. Standing long-cassette (36 $\times 14$ inch) anteroposterior (AP) radiographs taken from a distance of 2 meters by the same X-ray machine (Toshiba Model: BLR-1000A) which was calibrated as each small square on radiograph that is equal to $1 \mathrm{~cm}$ in the world, at preoperative and immediate postoperative (3-7 days) were evaluated to determine the changes of Cobb angle $(\theta)$, AVT, and the number of the vertebra in the curve to predict the 
difference of body height $(P \Delta H)$ by using a new formula and the simplified version of this formula $(S P \Delta H)$. The radiographs were scanned in a computer workstation by using a transparent media scanner (Mikrotek MRS-3200A3). Digital software (Canvas 9.0) was used for analyzing the measurements. To determine the pre- and post-operative AVT, the centroid of the apical vertebrae was located at the intersection of lines connecting the superior lateral corners of the vertebral body to the contralateral inferior lateral corners. The horizontal distance from this point to the C7 plumb line (a line dropped parallel to the film edge from the like-determined centroid of C7) was the AVT for the proximal and main thoracic curves. For thoracolumbar/lumbar curves, the horizontal distance was measured from the center sacral vertical line (CSVL) (i.e. a line drawn vertically from the midline of the sacrum) [5]. The $\Delta H, P \Delta H, S P \Delta H$, and the height loss by using the formula of Stokes [1] (Height loss $(\mathrm{mm})=1.0+0.066 *$ Cobb $+0.0084 *$ Cobb $*$ Cobb $)$ was noted using Microsoft Office Excel 2003. Statistical analysis was performed using SPSS (version13.0; Chicago, USA). One-way ANOVA and post-hoc test (Tukey HSD) was used to compare the parameters and Kruskal-Wallis test used to find the correlation with the prediction of height gain and type of scoliosis. The differences between real height gain $(\Delta H)$ and three predictive formulas $(P \Delta H, S P \Delta H$, and Stokes $\left.^{1}\right)$ were noted as $\Delta H-P \Delta H, \Delta H-S P \Delta H$ and $\Delta H$ Stokes $^{1}$ for statistic analysis. All surgeries were performed by the senior author. The average number of instrumented vertebrae was 9.67 (range 4-14) (Table 1).

In critically analyzing the data in Table 1 , the values that obtained by using Stokes ${ }^{1}$, formula differed from measured height gain $(\Delta H)$ in the majority of all cases after deformity correction. However. Stokes ${ }^{1}$ addressed only presumed height loss associated with a curve, without clinical validation. As also scoliosis is a three-dimensional deformity; correction maneuver that used during the surgery may change the distance between two vertebral bodies. To calculate the positive effect of intervertebral disc distance for each vertebra to height gain after correction; eleven patients (nine females and two males) were chosen from the study group that have $\leq 6.5^{\circ} \mathrm{Cobb}$ angle postoperatively (Table 2 ). The $k$ value was calculated by using the formula below:

$$
k=\frac{\text { mean value of }\left(\Delta H \text {-Stokes }{ }^{1}\right)}{N} .
$$

2.1. Evaluation of the Formula. When viewed in the coronal plane. the part of the preoperative curves is seen as in the form of $A B$, while we can measure the same segment as $A_{1} B_{1}$ after surgery (Figure 1 ).

Supposing that $A B$ curve is the arc of a circle with center $O$ and the radius of this circle is $R$, the corresponding angle to the arc is $\theta$, namely, Cobb angle (Figure 2). Cobb angle might be obtained by making a measurement on the plain radiographs of the patient.
The term for AVT was taken as $X_{0}$ in the observed patients. From the triangular and circular geometry, the chord of $A B$ and the arc-length of $A B$ can be written as

$$
\begin{gathered}
\text { the chord length of } A B=2 R \sin \left(\frac{\theta}{2}\right), \\
\text { the arc-length of } A B=\frac{2 \pi R}{360} \theta .
\end{gathered}
$$

Here, $\pi=3.14$ is the constant number. Providing that $100 \%$ correction is achieved after deformity correction, the geometrical contribution to the increase of height can be written as

$$
\begin{aligned}
& \text { the arc-length of } A B-\text { the chord length of } A B \\
& =\frac{2 \pi R}{360} \theta-2 R \sin \left(\frac{\theta}{2}\right) .
\end{aligned}
$$

On the other hand, due to taking the curved section as arc of circle, we can write the AVT as $X_{0}=R(1-\cos (\theta / 2))$. Using the formula the radius $R$ of circle can be expressed in terms of AVT; $X_{0}$ and of Cobb angle is $\theta$ :

$$
R=\frac{X_{0}}{1-\cos (\theta / 2)} .
$$

Consequently, the increase of height in a patient with $100 \%$ correction can be written as follows:

$$
\begin{aligned}
& \text { the arc-length of } A B-\text { the chord length of } A B \\
& =\frac{2 X_{0}}{1-\cos (\theta / 2)}\left(\frac{\pi}{360} \theta-\sin \left(\frac{\theta}{2}\right)\right) .
\end{aligned}
$$

The above formula has been obtained by assuming the spinal curvature forming an arc, considering the curvature as two-dimensional; although, the spinal curvature in scoliosis is three-dimensional. Therefore, the above formula might be an approximate formula considering only the Cobb angle.

If we take into account contributions from intervertebral disc distance, $P \Delta H^{1}$ might be used:

$$
P \Delta H^{1}=\frac{2 X_{0}}{1-\cos (\theta / 2)}\left(\frac{\pi}{360} \theta-\sin \left(\frac{\theta}{2}\right)\right)+a N .
$$

Here, $a$ is a constant parameter that was obtained from the 20 patients all have scoliotic curve. All patients were operated in our clinic and were not included in the current study. The preoperative and postoperative body heights of these patients were recorded, and the $a$ constant was found retrospectively by regression equation using the formula of $P \Delta H^{1}$ to be $2.3 \mathrm{~mm}$. The comparison of obtained $P \Delta H$, $S P \Delta H$, and $\Delta H$ is given in Table 1 . As it is frequently impossible to get $100 \%$ correction in Cobb angle and AVT; we recalculated the $P \Delta H^{1}$ for the patients that have residual deformities:

$$
P \Delta H=h 1-h+2.3\left(1-\frac{\theta_{2}}{\theta_{1}}\right) N,
$$

where

$$
\begin{aligned}
& h 1=\frac{2 X_{1}}{1-\cos \left(\theta_{1} / 2\right)}\left(\frac{\pi}{360} \theta_{1}-\sin \left(\frac{\theta_{1}}{2}\right)\right), \\
& h 2=\frac{2 X_{2}}{1-\cos \left(\theta_{2} / 2\right)}\left(\frac{\pi}{360} \theta_{2}-\sin \left(\frac{\theta_{2}}{2}\right)\right) .
\end{aligned}
$$




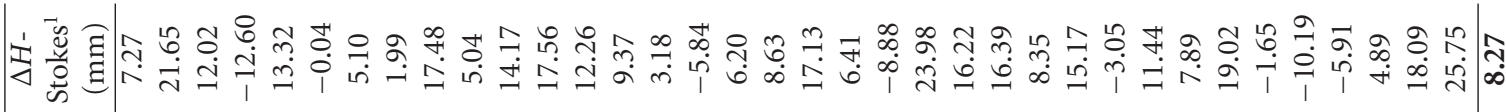

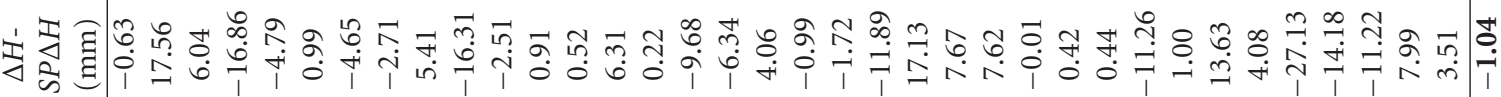

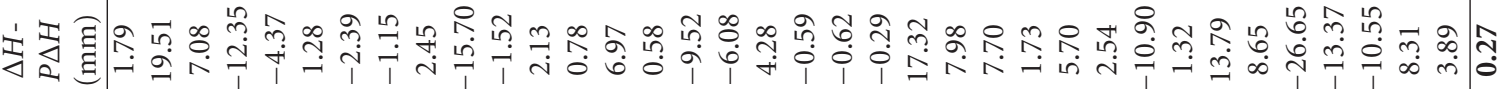

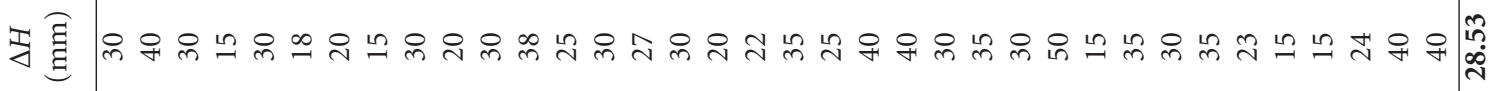

๘)

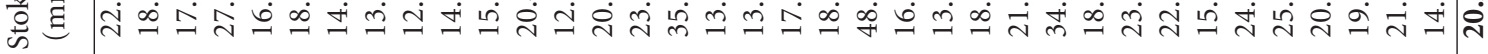

$\ddot{\sharp}$

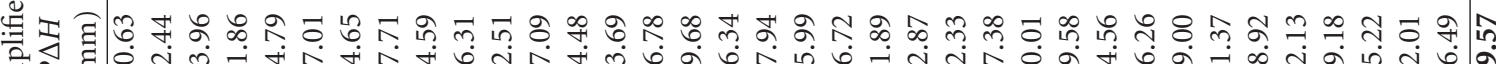
责

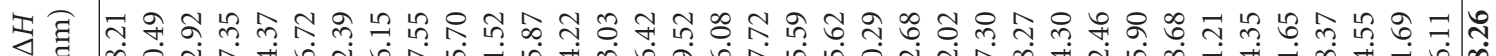
¿ है

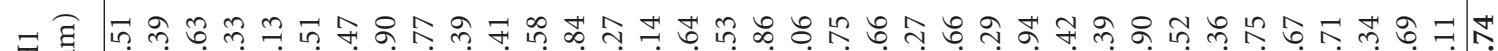
死 毒 ₹ 焉

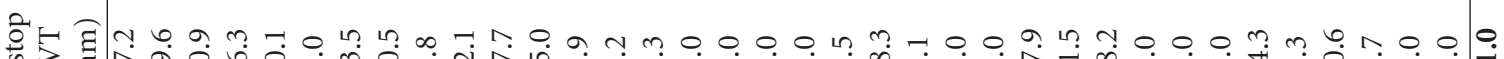
क्ष 产

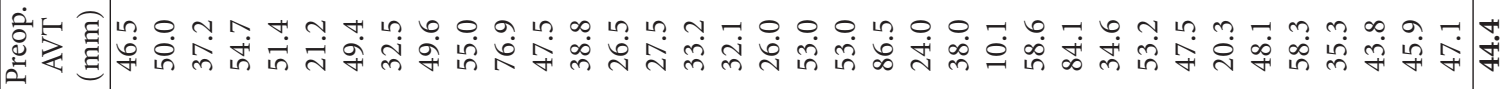

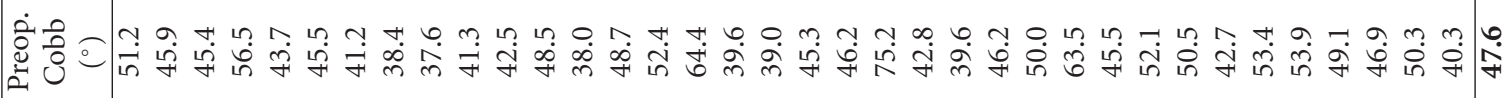

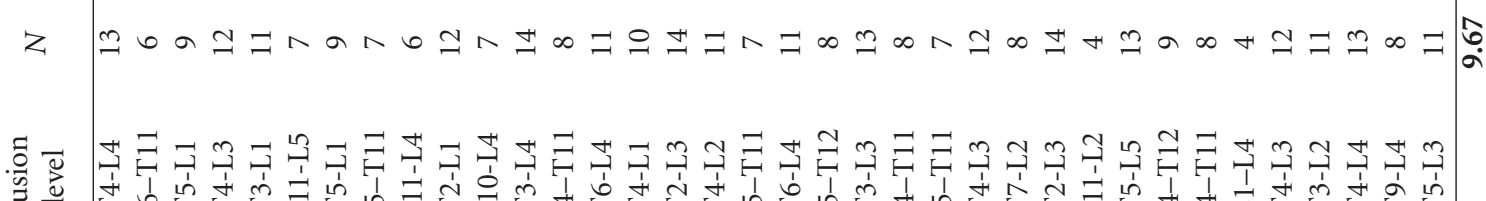

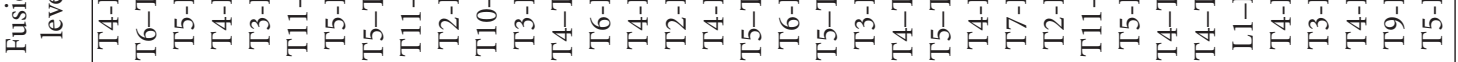

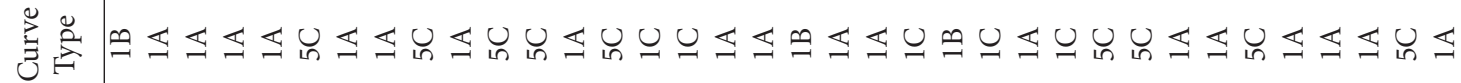

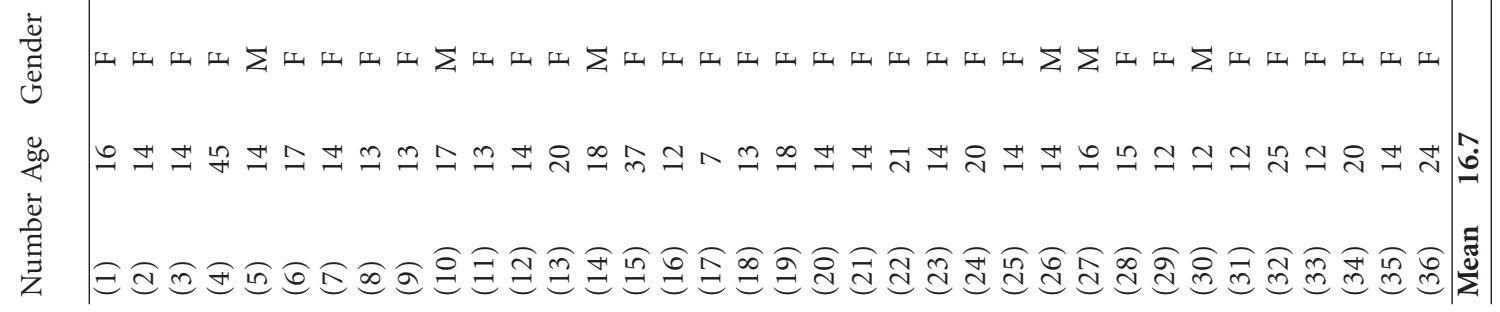




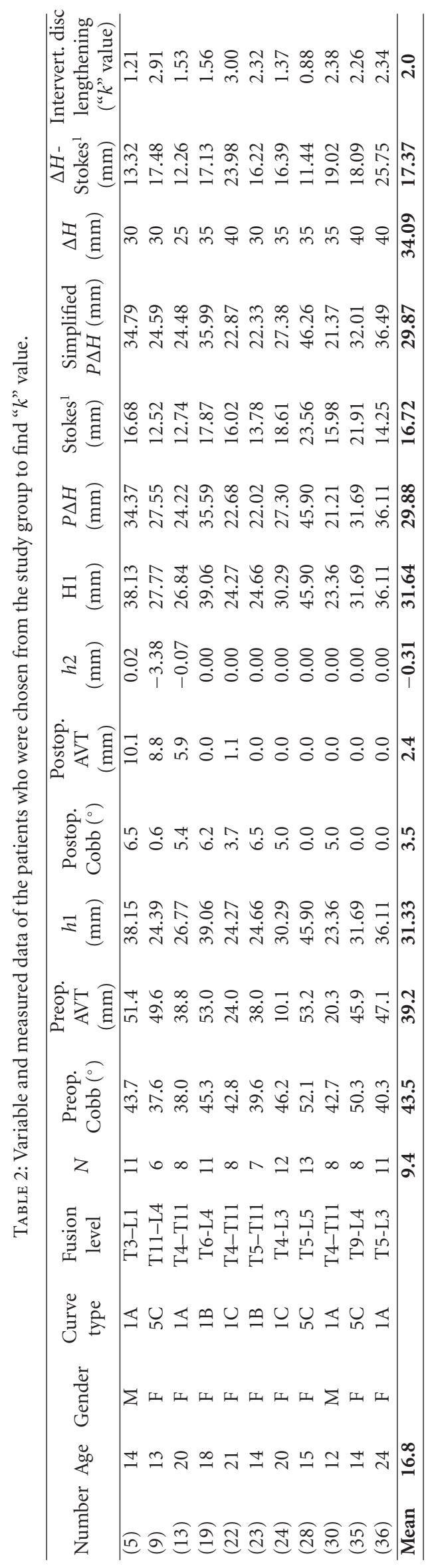




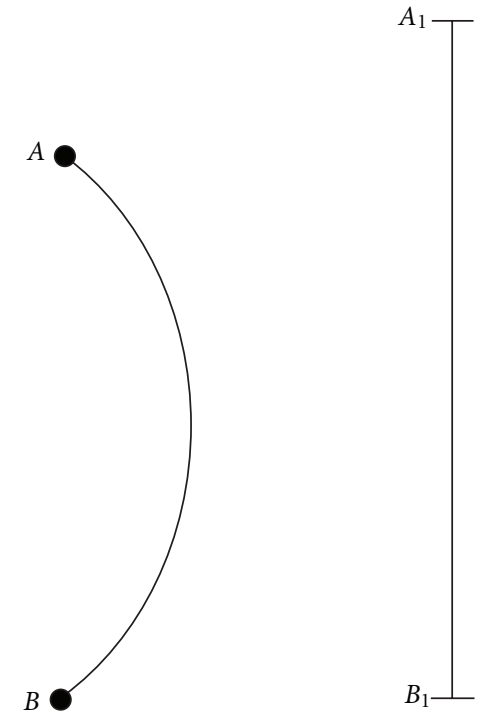

FIGURE 1: $|A B|$ is the part of the preoperative spinal length. $\left|A_{1} B_{1}\right|$ is the postoperative view of the same segment.

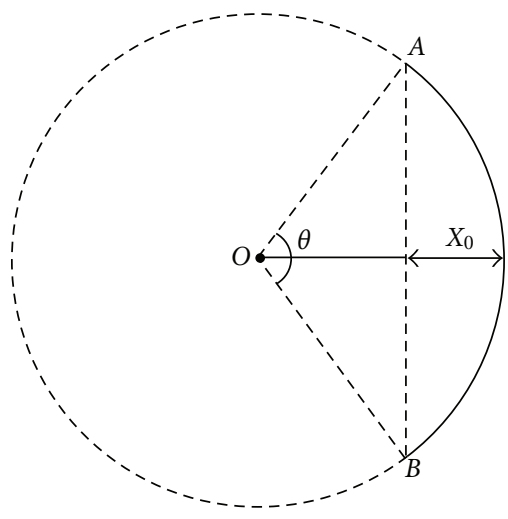

Figure 2: " $A B$ " curve is the arc of a circle with center " $O$," and the radius of this circle is $R$, the corresponding angle to the arc is $\theta$ (Cobb angle), while the AVT is $X_{0}$.

And the final form of the formula is;

$$
\begin{aligned}
P \Delta H= & \frac{2 X_{1}}{1-\cos \left(\theta_{1} / 2\right)}\left(\frac{\pi}{360} \theta_{1}-\sin \left(\frac{\theta_{1}}{2}\right)\right) \\
& -\frac{2 X_{2}}{1-\cos \left(\theta_{2} / 2\right)}\left(\frac{\pi}{360} \theta_{2}-\sin \left(\frac{\theta_{2}}{2}\right)\right) \\
& +2.3\left(1-\frac{\theta_{2}}{\theta_{1}}\right) N .
\end{aligned}
$$

In our predictive formula, $\theta_{1}$ is preoperative Cobb angle. $X_{1}$ is preoperative AVT while $\theta_{2}$ and $X_{2}$ is postoperative Cobb angle and AVT, $N$ is the number of vertebra that was instrumented.

Although this formula contains all variables which may contribute to height gain, it is too complex and unusable in daily practice. In order to use this formula for practical calculation, we rewrite to simplify this expression using Taylor series method. The Taylor series of a function $f(x)$ that is infinitely differentiable in a neighborhood of a real number $b$ is the power series. As usually Cobb angles in scoliosis patients varies approximately between $35^{\circ}$ and $75^{\circ}$ we applied Taylor expansion to above formula as neighborhood of Cobb angle of $45^{\circ}$. As seen, our formula includes trigonometric functions $\sin \theta$ and $\cos \theta$. For example, Taylor series of $\sin \theta$ is as follows:

$$
\begin{aligned}
\sin \theta= & \sin \theta_{0}+\left(\theta-\theta_{0}\right) \cos \theta_{0} \\
& -\frac{1}{2}\left(\theta-\theta_{0}\right)^{2} \sin \theta_{0}-\frac{1}{6}\left(\theta-\theta_{0}\right)^{3} \cos \theta_{0} .
\end{aligned}
$$

First, a few terms of this series are sufficient in our calculations. Using above expansion of $\sin \theta$ and similar expression for $\cos \theta$ after some simplifications, we obtain very simple expression for height gain:

$$
S P \Delta H=0.0059 X_{1} \theta_{1}+2.3\left(1-\frac{\theta_{2}}{\theta_{1}}\right) N,
$$

$\theta_{1}$ : preoperative Cobb angle, $X_{1}$ : preoperative AVT, $\theta_{2}$ : postoperative Cobb angle, $N$ : the number of instrumented vertebra.

\section{Results}

The mean preoperative Cobb angle of $47.6^{\circ}$ (range, $37.6^{\circ}-$ $75.2^{\circ}$ ) was corrected to $12.4^{\circ}$ (range, $0^{\circ}-41.2^{\circ}$ ). The mean preoperative and postoperative AVT was $44.4 \mathrm{~mm}$ (range, $10.1 \mathrm{~mm}-86.5 \mathrm{~mm}$ ) and $11.0 \mathrm{~mm}$ (range, $0 \mathrm{~mm}-48.3 \mathrm{~mm}$ ), respectively; the mean $\Delta H$ was $28.53 \mathrm{~mm}$ (range, $15 \mathrm{~mm}-$ $50 \mathrm{~mm}$ ). The mean $P \Delta H$ that measured by using new formula was $28.26 \mathrm{~mm}$ (range, $12.46 \mathrm{~mm}-45.9 \mathrm{~mm}$ ). The mean $S P \Delta H$ was $29.57 \mathrm{~mm}$ (range, $14.56 \mathrm{~mm}-51.89 \mathrm{~mm}$ ). The mean value of the height gain that was noted using the Stokes $^{1}$ formula was $20.26 \mathrm{~mm}$ (range, $12.52 \mathrm{~mm}-$ $48.88 \mathrm{~mm}$ ). The mean $\Delta H-P \Delta H$ was $0.27 \mathrm{~mm}$ (standard deviation $=9.26$ ), and the mean $\Delta H-S P \Delta H$ was $-1.04 \mathrm{~mm}$ ( standard deviation $=9.51)$, while $\Delta H$-Stokes ${ }^{1}$ was $8.27 \mathrm{~mm}$ ( standard deviation $=9.77)$. Although there were significant differences between $\Delta H$-Stokes ${ }^{1}$ with either $\Delta H-P \Delta H(P=$ $0.002)$ or $\Delta H-S P \Delta H(P=0.000)$, there was no significant difference between $\Delta H-P \Delta H$ and $\Delta H-S P \Delta H(P=0.829)$. There was no significant correlation between curve type and three predictive formulas $(P=0.265$ for $\Delta H-P \Delta H . P=$ 0.279 for $\Delta H-S P \Delta H$, and $P=0.561$ for $\Delta H$-Stokes $\left.{ }^{1}\right)$. The mean $k$ value was $(2 \pm 0.70) \mathrm{mm}$ (Table 2$)$.

\section{Discussion}

In the early conservative treatment periods of 1950-1960s, the height gain measurement in scoliosis patients was aimed to assess vital capacity [2.6.7]. Archer and Dickson mentioned about spinal height gain due to kyphoscoliosis correction in 1985 [6]. Ylikoski was the first to suggest a formula predicting height loss in untreated scoliotic patients [4]. Stokes has recently suggested a new formula to determine height loss in idiopathic curves [1]. None of these previous formulas on height loss attributed to the deformity, however, has had clinical correlation. 


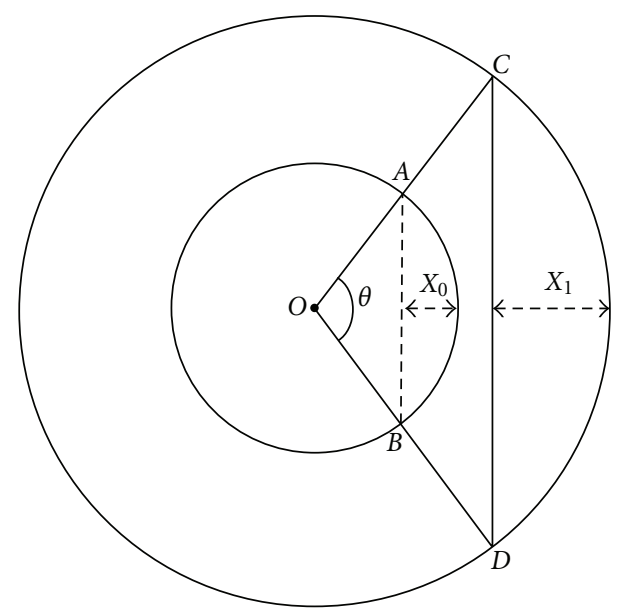

Figure 3: $|A B| \neq|C D|$ and $X_{0} \neq X_{1}$, although these two arcs have the same Cobb angle $(\theta)$.

Our hypothesis like that of Brookenthal [3] assumes a circular geometry of the scoliotic curve. Stokes assumed that spinal height occurs only as a result of altered curvature without alteration in disc height [1]. While the relationship between height loss and Cobb angle was determined by regression analyses, it is obvious that for the same Cobb angle the spinal length and height may change with respect to AVT and number of instrumented vertebrae that has not been taken into consideration by Stokes [1] (Figure 3).

Scoliotic curvature of the spine results from a combination of disc and vertebral wedging. Our analyses in clinical setting showed Stokes' formula to underestimate height gain after deformity correction. To find out contribution of each disc space to height gain, we have used the formula to find " $k$ " value: $k=$ mean value of $\left(\Delta H\right.$-Stokes $\left.{ }^{1}\right) / N$. The " $k$ " value being equal to " $a$ " constant in our regression analyses shows the reliability of the " $k$ " value. This suggests that the surgery increased disc height while reducing the Cobb angle.

Stokes quantified the spinal deformity by the average of two Cobb angles in double curves whether or not both were considered structural [1]. Our analysis does not compromise sufficient number of patients having double curves to comment on height gain in such patients. Mean errors ranging from $1.7^{\circ}$ to $6.5^{\circ}$ in both manual and digital measure of Cobb angle [7-11], two-dimensional measurement of a three-dimensional deformity, possible erroneous height measurements in clinical setting are the main limitations of this study. However, our application of the formula to scoliosis patients suggests being applicable.

In idiopathic scoliosis, the convex sides of bodies. Discs, pedicles, intervertebral foramina, and lamina are greater than the depths of the same structures on the concave side [12]. During posterior instrumented deformity correction by rod derotation, translation maneuver of posterior elements on the concave side is lengthened. Thus, the concave-sided rod gets shorter than the measured curve length. The present study was motivated by the need to estimate percent curve correction intraoperatively by the relative rod shortening on the concave side using our new simplified predictive mathematical formula:

$$
S P \Delta H=0.0059 X_{1} \theta_{1}+2.3\left(1-\frac{\theta_{2}}{\theta_{1}}\right) N .
$$

To illustrate the results, an individual patient is presented with a single thoracic curve of $50^{\circ}$ Cobb angle and $30 \mathrm{~mm}$ AVT. We have planned eight-vertebra instrumentation and $70 \%$ correction with derotation maneuver. Therefore, final Cobb angle must be $15^{\circ}$ postoperatively. Using our simplified formula:

$$
S P \Delta H=0.0059 * 30 * 50+2.3\left(1-\frac{15}{50}\right) 8 .
$$

$S P \Delta H=21.73 \mathrm{~mm}$; likewise, we have to cut our concave sided rod $21.73 \mathrm{~mm}$ longer than the measured spinal height intraoperatively to achieve the planned $70 \%$ correction. It is interesting to note that for two right thoracic scoliotic deformities with equal Cobb angle spinal height may change significantly with even a single change in the number instrumented vertebra. Estimating percent curve correction intraoperatively by rod length, however, is hindered by many factors which if solved may in future serve as a tool to optimize instrumentation. If we quantitate curve correction, it may also help to prevent trunk imbalance in the frontal plane that is sometimes encountered with posterior derotational systems. Using the formulas from the measurements in the intraoperative X-rays may solve many of the problems due to patient positioning, anesthesia reducing spine stiffness, and calibration problems causing sizable changes in the exact measure of spinal height by preoperative radiographs.

Definition of the scoliotic deformity as an arc is an oversimplification of the three-dimensional deformity. Relying on two-dimensional measurement as well as not considering the compensatory curves are the main shortcomings of our study. Also the current formula may be more appreciable to younger patients with relatively small curves without vertebral body deformation.

\section{Conclusion}

From the findings in this study, it would appear that AVT. number of instrumented vertebra and " $k$ " value of $(2 \pm 0.70)$ $\mathrm{mm}$ for each instrumented disc space offers a significant additional parameter to Cobb angle to achieve an almost perfect prediction of postoperative height gain in idiopathic scoliosis.

\section{Acknowledgement}

The authors particularly emphasize their gratitude to Professor Ian Stokes. Ph.D, (University of Vermont) for his "helpful comments and suggestions" during the preparation of the paper. 


\section{References}

[1] I. A. F. Stokes, "Stature and growth compensation for spinal curvature," Studies in Health Technology and Informatics, vol. 140, pp. 48-51, 2008.

[2] J. Bjure and A. Nachemson, "Non-treated scoliosis," Clinical Orthopaedics and Related Research, vol. 93, pp. 44-52, 1973.

[3] K. R. Brookenthal, "Loss of height in scoliosis due to degree of curvature," in Proceedings of the 15th Conference of Scoliosis Research Society, 2002.

[4] M. Ylikoski, "Height of girls with adolescent idiopathic scoliosis," European Spine Journal, vol. 12, no. 3, pp. 288-291, 2003.

[5] T. R. Kuklo, B. K. Potter, T. M. Schroeder, and M. F. O'Brien, "Comparison of manual and digital measurements in adolescent idiopathic scoliosis," Spine, vol. 31, no. 11, pp. 1240-1246, 2006.

[6] I. A. Archer and R. A. Dickson, "Stature and idiopathic scoliosis: a prospective study," Journal of Bone and Joint Surgery B, vol. 67, no. 2, pp. 185-188, 1985.

[7] C. J. Adam, M. T. Izatt, J. R. Harvey, and G. N. Askin, "Variability in Cobb angle measurements using reformatted computerized tomography scans," Spine, vol. 30, no. 14, pp. 1664-1669, 2005.

[8] A. De Carvalho, R. Vialle, L. Thomsen et al., "Reliability analysis for manual measurement of coronal plane deformity in adolescent scoliosis. Are 30-90 cm plain films better than digitized small films?" European Spine Journal, vol. 16, no. 10, pp. 1615-1620, 2007.

[9] K. G. Shea, P. M. Stevens, M. Nelson, J. T. Smith, K. S. Masters, and S. Yandow, "A comparison of manual versus computer-assisted radiographic measurement: intraobserver measurement variability for Cobb angles," Spine, vol. 23, no. 5, pp. 551-555, 1998.

[10] I. A. F. Stokes and D. D. Aronsson, "Computer-assisted algorithms improve reliability of king classification and cobb angle measurement of scoliosis," Spine, vol. 31, no. 6, pp. 665670, 2006.

[11] B. P. D. Wills, J. D. Auerbach, X. Zhu et al., "Comparison of Cobb angle measurement of scoliosis radiographs with preselected end vertebrae: traditional versus digital acquisition," Spine, vol. 32, no. 1, pp. 98-105, 2007.

[12] R. ROAF, "Vertebral growth and its mechanical control.," The Journal of Bone \& Joint Surgery. British, vol. 42, pp. 40-59, 1960. 


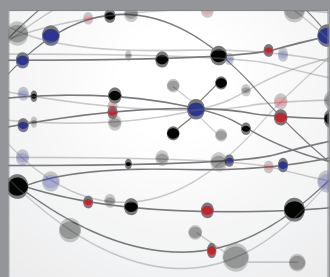

The Scientific World Journal
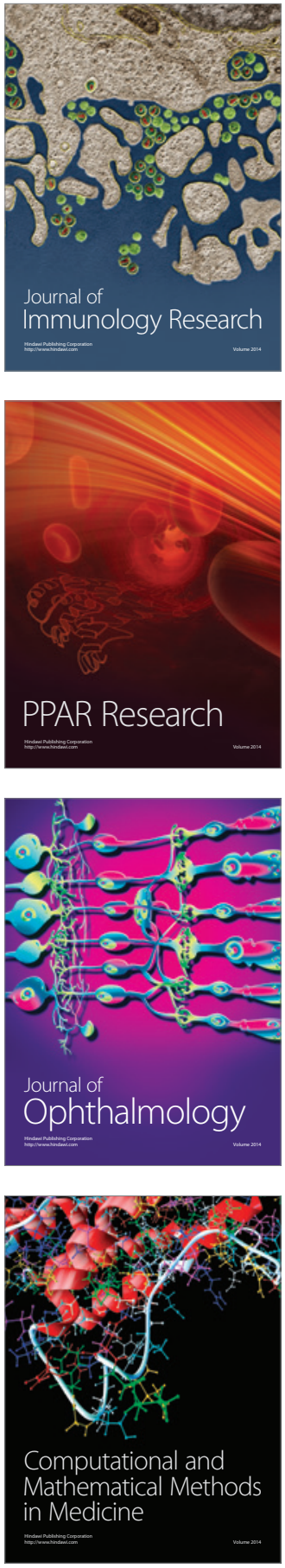

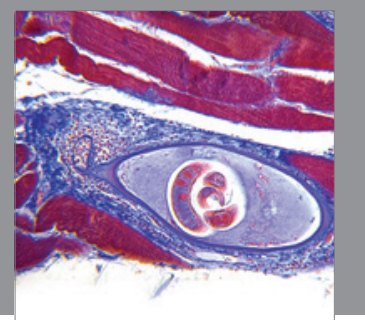

Gastroenterology

Research and Practice
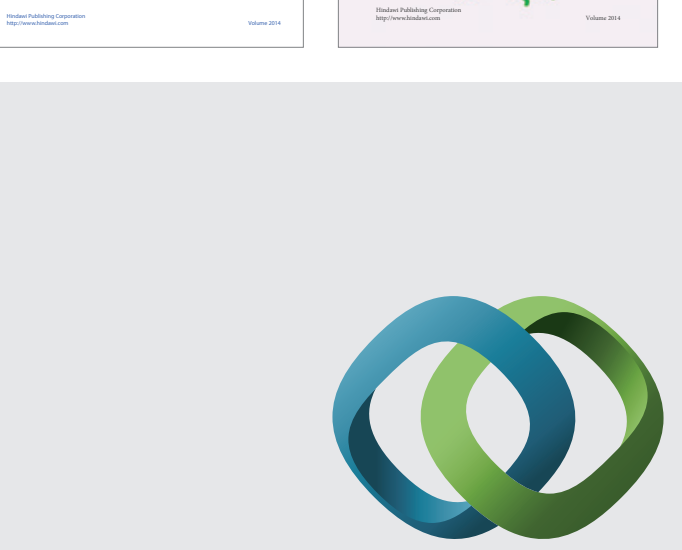

\section{Hindawi}

Submit your manuscripts at

http://www.hindawi.com
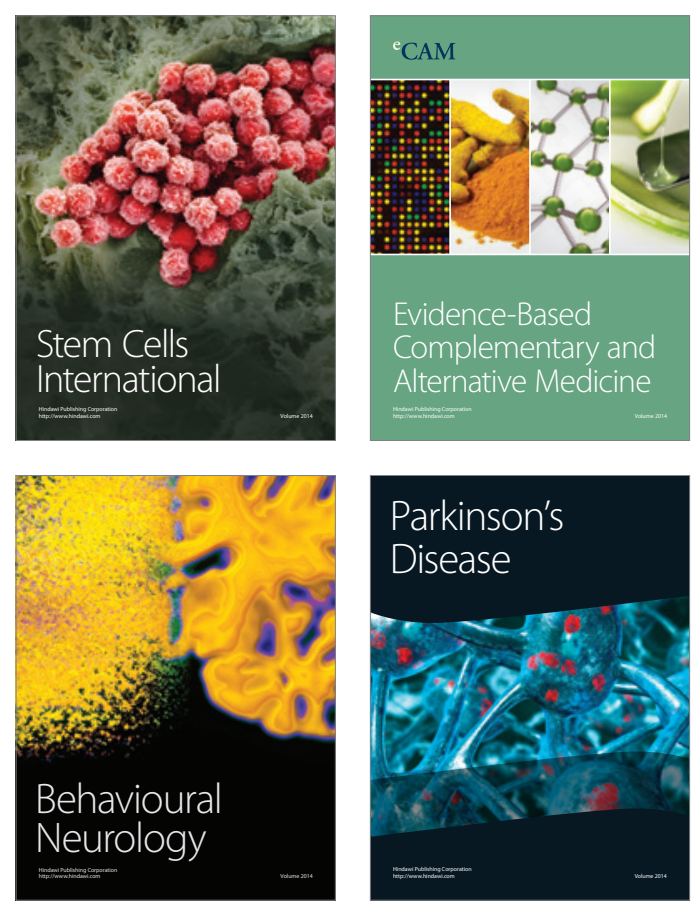

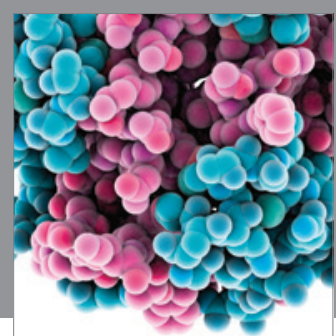

Journal of
Diabetes Research

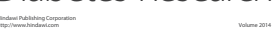

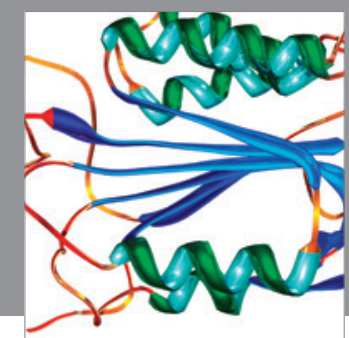

Disease Markers
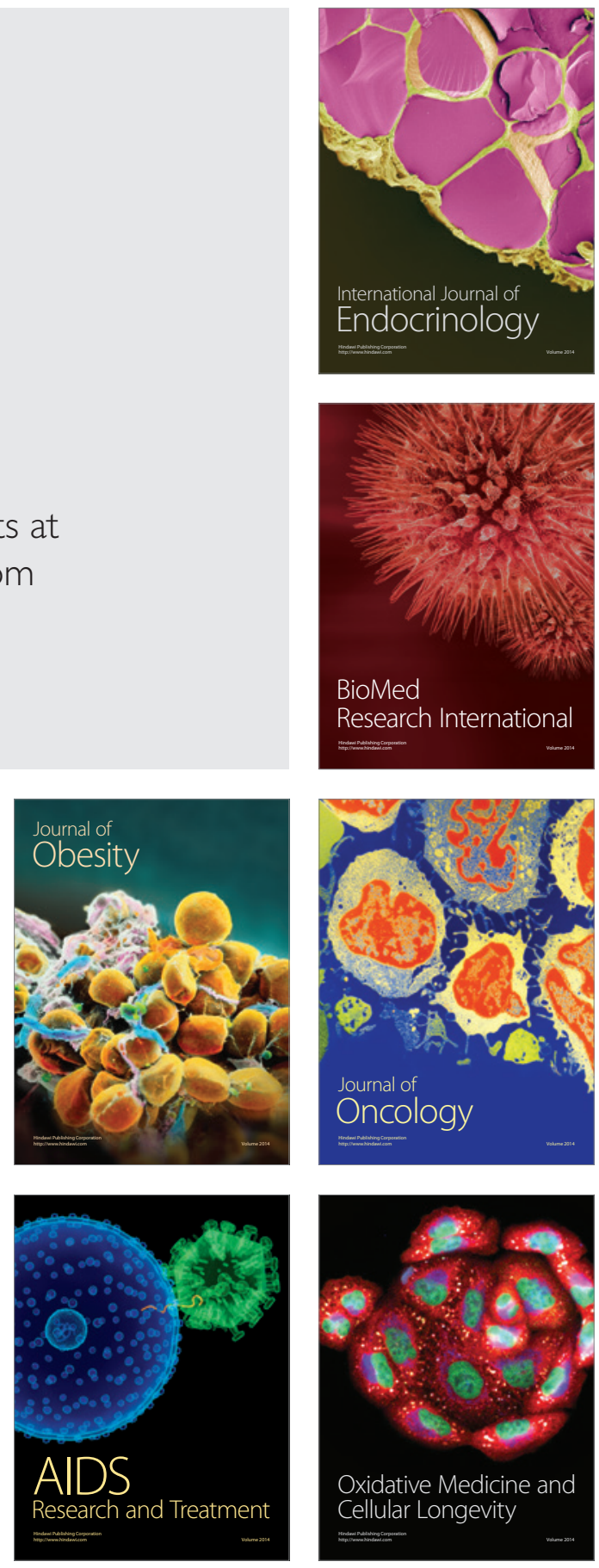